\title{
Ethnic differences in BMI, subcutaneous fat, and serum leptin levels during and after pregnancy and risk of gestational diabetes
}

\author{
Christine Sommer ${ }^{1,2}$, Anne K Jenum ${ }^{3,4}$, Christin W Waage ${ }^{1,3}$, Kjersti Mørkrid $^{5}$, \\ Line Sletner ${ }^{6}$ and Kåre I Birkeland ${ }^{1,2}$ \\ ${ }^{1}$ Department of Endocrinology, Morbid Obesity and Preventive Medicine, Oslo University Hospital, \\ PO Box 4959 Nydalen, N-0424 Oslo, Norway, ${ }^{2}$ Faculty of Medicine, Institute of Clinical Medicine and \\ ${ }^{3}$ Department of General Practice, Faculty of Medicine, Institute of Health and Society, University of Oslo, Oslo, \\ Norway, ${ }^{4}$ Faculty of Health Sciences, Oslo and Akershus University College of Applied Sciences, Oslo, Norway, \\ ${ }^{5}$ Department of International Public Health, Norwegian Institute of Public Health, Oslo, Norway and \\ ${ }^{6}$ Department of Child and Adolescents Medicine, Akershus University Hospital, Lørenskog, Norway
}

Correspondence should be addressed to C Sommer Email

christine.sommer@ medisin.uio.no

\begin{abstract}
Objective: To explore the differences between Europeans and South Asians in BMI, subcutaneous fat, and serum leptin (s-leptin) levels during and after pregnancy and their relationship with gestational diabetes (GDM).

Design: Multi-ethnic population-based cohort study, whereof 353 Europeans (93.1\% of the included) and 190 South Asians $(95.0 \%$ of the included).

Methods: S-leptin, BMI, and subcutaneous fat (sum of triceps, subscapular, and suprailiac skinfolds) were measured at 14 and 28 weeks of gestation, and 14 weeks after delivery. GDM was diagnosed with the WHO criteria 2013.

Results: South Asians had similar thickness of the triceps and suprailiac skinfolds, thicker subscapular skinfold, and higher s-leptin than Europeans in early pregnancy, despite lower BMI. South Asians retained more subcutaneous fat (mean $(95 \% \mathrm{Cl}) 10.0(7.4-12.7) \mathrm{mm}$ vs $3.8(1.9-5.8) \mathrm{mm})$ and BMI $\left(1.5(1.2-1.8) \mathrm{kg} / \mathrm{m}^{2}\right.$ vs $0.1(-0.1$ to 0.3$\left.) \mathrm{kg} / \mathrm{m}^{2}\right)$ than Europeans 14 weeks after delivery and s-leptin decreased less in South Asians than Europeans $(-0.13(-0.27$ to -0.00$) \mu \mathrm{g} / \mathrm{lvs}-0.47$ $(-0.57$ to -0.37$) \mu \mathrm{g} / \mathrm{l}, P<0.001$ for all). The prevalence of GDM was $23.8 \%(n=84)$ in Europeans and $42.6 \%(n=81)$ in South Asians. BMI, subcutaneous fat, and s-leptin were all positively associated with GDM, also after adjustment for covariates. Conclusions: The relatively high amounts of subcutaneous fat and s-leptin in South Asians in early pregnancy contributed to their increased risk of GDM. South Asians retained more weight and subcutaneous fat after delivery, potentially increasing their risk of adiposity and GDM in future pregnancies.
\end{abstract}

\section{Introduction}

Obesity and diabetes constitute worldwide threats to the public health (1) and to health care systems and economies (2). South Asian migrants to Europe have a high prevalence of type 2 diabetes (3) and gestational diabetes (GDM) (4). GDM is defined as first recognition of hyperglycemia during pregnancy (5). GDM gives a sevenfold higher risk of developing type 2 diabetes in the future (6), is associated
(C) 2015 European Society of Endocrinology Printed in Great Britain with several pregnancy complications $(7,8)$, and probably increases the risk of later obesity and type 2 diabetes in the offspring (9). Maternal obesity increases the risk of GDM, large for gestational age babies (10) and even of fetal death, stillbirth, and infant death (11).

The adverse metabolic consequences of obesity, such as insulin resistance and diabetes, are related to the 
accumulation of fat (12). Serum leptin (s-leptin) levels reflect the proportion of adipose tissue in the body (13), is highly correlated with fat mass (14), and correlates particularly with subcutaneous fat in women $(14,15)$. Several studies have observed higher s-leptin levels in individuals of South Asian than European descent for the same BMI $(16,17)$, suggesting that BMI may be a poor indicator of adiposity in South Asians. Leptin levels increase in pregnancy, due to production by the placenta, and decline rapidly after delivery (18). Thus, subcutaneous fat and leptin may be better indicators of adiposity in a multi-ethnic population of pregnant women than body weight and BMI, and may account for some of the higher GDM risk observed in South Asians. To our knowledge, ethnic differences in subcutaneous fat and leptin levels during and after pregnancy and their relationship with GDM risk have not yet been investigated.

Our objective was to explore the differences between European and South Asian women in BMI, subcutaneous fat, and s-leptin levels during and after pregnancy and their relationships with GDM.

\section{Subjects and methods}

Details of the study methods have been described previously (19). The STORK Groruddalen study is a population-based cohort study of healthy pregnant women attending child health clinics for antenatal care in three administrative city districts in Groruddalen, Oslo, Norway, from May 2008 to May 2010. General practitioners were asked to refer pregnant women to the child health clinics early in pregnancy. Women were eligible if they i) lived in the study districts, ii) planned to give birth at one of two study hospitals, iii) were $<20$ weeks pregnant, and iv) could communicate in Norwegian or any of the eight translated languages. Women with pregestational diabetes or in need of intensive hospital follow-up during pregnancy were excluded. The participation rate was $81.5 \%$ among Europeans and 73.0\% among South Asians.

The study was approved by The Regional Ethics Committee and The Norwegian Data Inspectorate. A written consent was obtained for all the participants after full explanation of the purpose and procedures used.

\section{Background data}

Information about maternal age, parity, and ethnic origin was collected through interviewer-administered questionnaires at 14 weeks of gestation. Parity was dichotomized into nulliparous and parous, referring to status before the current pregnancy. Ethnicity was defined as country of birth or participant's mother's country of birth if the participant's mother was born outside of Europe or other western countries. Three women born in North America were categorized as Europeans. In this substudy, we included all Europeans ( $82 \%$ Norwegians) and South Asians (63\% Pakistanis and 31\% Sri Lankans). Family history of diabetes was defined as having a firstdegree relative with diabetes.

\section{Anthropometrics}

Height was measured with a fixed stadiometer to the nearest $0.1 \mathrm{~cm}$ at 14 weeks of gestation, body weight with a calibrated digital scale with light clothing and without shoes at 14 and 28 weeks of gestation, and 14 weeks after delivery. BMI was calculated with height measured at 14 weeks of gestation and weight at the respective visits. Prepregnancy BMI was based on self-reported prepregnant body weight.

Subcutaneous fat was measured with a skinfold caliper, to the nearest $1 \mathrm{~mm}$, at the triceps, subscapular, and suprailiac sites, at 14 and 28 weeks of gestation and 14 weeks after delivery $(20,21)$. Each skinfold was measured twice and the mean value was used. Sum of skinfolds, a proxy for overall subcutaneous fat (22), was calculated by summarizing the three skinfold sites. Inter-rater variability for maternal skinfolds, expressed as \%technical error of measurement, ranged from 5 to $21 \%$ between study personnel and the reference midwife (21). Intra-rater variability was $<5 \%$ for all measurements (21).

\section{Metabolic variables and GDM}

Blood was collected at 14 and 28 weeks of gestation and 14 weeks after delivery. Fasting C-peptide and insulin were measured at the Hormone Laboratory, Oslo University Hospital, by non-competitive immunofluorometric assays (DELFIA, PerkinElmer Life Sciences, Wallac Oy, Turku, Finland). HbA1c was analyzed with HPLC (Tosoh G8, Tosoh Corporation, Tokyo, Japan). S-leptin was analyzed with the Luminex XMAP technology (Millipore Corporation, Billerica, MA, USA). A standard $75 \mathrm{~g}$ oral glucose tolerance test (OGTT) was performed at 28 weeks of gestation with fasting and 2-h plasma glucose analyzed on-site in venous EDTA blood samples (HemoCue, Angelholm, Sweden) (4). GDM was defined by the WHO 2013 criteria (23) (fasting glucose $\geq 5.1 \mathrm{mmol} / \mathrm{l}$ or 2 -h glucose $\geq 8.5 \mathrm{mmol} / \mathrm{l}$ ) and 1 -h glucose was not available (4). 
Excluded due to ethnic origin $(n=244)$

379 Europeans 200 South Asians

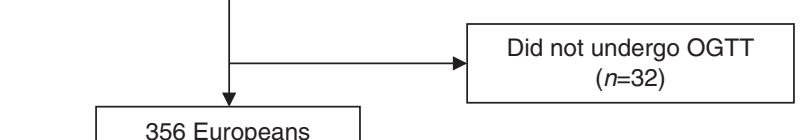

191 Europeans

191 South Asians

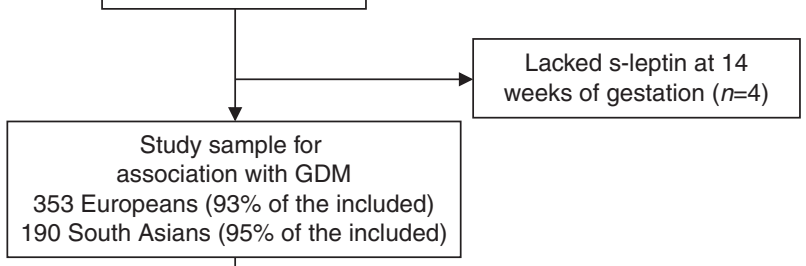

Did not attend at visit 14 weeks postpartum $(n=76)$

\section{Figure 1}

Flow chart of the cohort. OGTT, oral glucose tolerance test.

\section{Statistical analysis}

Continuous variables were expressed as mean \pm s.D. if normally distributed, median (interquartile range) if not normally distributed, or number (\%) if categorical. Estimated means adjusted for covariates were presented as mean $(95 \% \mathrm{CI})$, and results from the logistic regression analyses were presented as OR (95\% CI). Level of significance was set at $P<0.05$. S-leptin at each time point was transformed by square root to obtain normal distribution before analyses and subsequently transformed back for reporting purposes. The change variables of s-leptin approached normal distribution. For simple comparisons of continuous variables, we used $t$-test or Mann-Whitney $U$ test as appropriate and $\chi^{2}$ test to test differences in proportions. To test overall change between visits, we performed paired samples $t$-test. We performed multivariate general linear models (GLM) to explore ethnic difference in the changes during pregnancy and after delivery. The changes from 14 to 28 weeks of gestation were adjusted for weeks of gestation at inclusion, weeks between the two measurements, age, and parity, while changes from 14 weeks of gestation to 14 weeks after delivery were adjusted for weeks of gestation at inclusion, week after delivery, age, and parity. To explore ethnic differences in body composition at 14 weeks of gestation in nulliparous vs parous, we performed multivariate GLM across ethnic origin separately for nulliparous and parous and adjusted for weeks of gestation at inclusion and age.

We performed logistic regression analyses separately for each explanatory variable to explore the association between early pregnancy BMI, sum of skinfolds or s-leptin, and GDM. The logistic regression analyses were adjusted for weeks of gestation at inclusion, age, parity, and ethnic origin. All statistical analyses were performed using SPSS IBM Statistics 21.

\section{Sample flow}

Of the 823 women participating in the STORK Groruddalen study, we included all women with European $(n=379)$ and South Asian $(n=200)$ origin (Fig. 1). We found no differences in baseline characteristics, listed in Table 1, between those lacking OGTT or s-leptin and the included South Asians and Europeans (data not shown). When exploring ethnic differences in BMI, skinfolds and s-leptin during and after pregnancy, we excluded 76 women who did not attend the visit at 14 weeks after delivery (Fig. 1). Among the women who attended 14 weeks after delivery, there was a higher proportion of nulliparous than parous (referring to their status before the current pregnancy; $49.7 \%$ vs $38.1 \%, P=0.003$ ) and they had slightly lower prepregnant BMI $\left(25.4 \mathrm{~kg} / \mathrm{m}^{2}\right.$ vs $\left.24.2 \mathrm{~kg} / \mathrm{m}^{2}, P=0.011\right)$ than those who did not re-attend.

Table 1 Characteristics of the sample at 15 weeks of gestation across ethnic origin. Data are expressed as mean \pm s.D., $n(\%)$ or median (interquartile range).

\begin{tabular}{|c|c|c|c|}
\hline & Europe $(n=353)$ & $\begin{array}{l}\text { South Asia } \\
\quad(n=190)\end{array}$ & $\boldsymbol{P}^{\mathrm{a}}$ \\
\hline Age (years) & $30.1 \pm 4.5$ & $28.2 \pm 4.5$ & $<0.001$ \\
\hline Nulliparous, $n(\%)$ & $188(53)$ & $79(42)$ & 0.013 \\
\hline Height (cm) & $167 \pm 6$ & $160 \pm 6$ & $<0.001$ \\
\hline Body weight $(\mathrm{kg})$ & $70.4 \pm 13.7$ & $62.6 \pm 11.5$ & $<0.001$ \\
\hline $\begin{array}{l}\text { Prepregnancy BMI, } \\
\text { self-reported } \\
\left(\mathrm{kg} / \mathrm{m}^{2}\right)\end{array}$ & $24.5 \pm 4.8$ & $23.7 \pm 4.1$ & 0.041 \\
\hline HbA1c (\%) & $5.1 \pm 0.2$ & $5.2 \pm 0.3$ & $<0.001$ \\
\hline $\mathrm{HbA} 1 \mathrm{c}(\mathrm{mmol} / \mathrm{mol})$ & $32 \pm 2.2$ & $33 \pm 3.3$ & \\
\hline Insulin (pmol/l) & $32.5(23.0-48.0)$ & $52.5(36.3-78.8)$ & $<0.001$ \\
\hline C-peptide (pmol/l) & 478 (384-612) & 596 (469-804) & $<0.001$ \\
\hline $\begin{array}{l}\text { Family history of } \\
\text { diabetes, } n(\%)\end{array}$ & $48(14.2)$ & $88(47.8)$ & $<0.001$ \\
\hline
\end{tabular}

${ }^{a} t$-test if mean \pm s.D. is presented, Mann-Whitney $U$ test if median (interquartile range), and $\chi^{2}$ if $n(\%)$ is presented. 


\section{Results}

South Asians were younger and had higher parity than Europeans (Table 1). South Asians had lower body height and weight at 14 weeks of gestation and lower prepregnancy BMI than Europeans. South Asians had higher HbA1c, C-peptide, and insulin levels than Europeans at 14 weeks of gestation (Table 1). Although the median week of inclusion was 14 for both South Asians and Europeans, the interquartile range showed that South Asians were included $\sim 1$ week later than Europeans (14 (12-16) vs 14 (13-17) weeks of gestation for Europeans and South Asians respectively, $P=0.001$ ). At 14 weeks of gestation, we found no ethnic differences in triceps and suprailiac skinfolds, while the subscapular skinfolds were thicker and s-leptin higher in South Asians than Europeans, despite lower BMI (Table 2).

\section{Status at 28 weeks of gestation and 14 weeks after delivery}

At 28 weeks of gestation, South Asians still had lower BMI, but larger triceps, subscapular, and sum of skinfolds (Table 2). South Asians also had higher s-leptin levels than Europeans. BMI, skinfolds, and s-leptin increased from 14 to 28 weeks of gestation $(P<0.001$ for all). We found no difference between South Asians and Europeans in the increase of BMI or skinfolds (Table 2), neither after adjustments for differences in weeks of gestation at inclusion, number of weeks between the two measurements, age, and parity. S-leptin increased more in South Asians than Europeans from 14 to 28 weeks of gestation (Table 2), also after adjustment for differences in weeks of gestation at inclusion, number of weeks between the two measurements, age, and parity.

At 14 weeks after delivery, South Asians had reached the same BMI as Europeans, but South Asians had higher levels of all skinfold measurements (Table 2 and Fig. 2). As observed in early pregnancy, South Asians still had higher s-leptin levels than Europeans (Table 2 and Fig. 2). From early pregnancy to 14 weeks after delivery, after adjustment for weeks of gestation at inclusion, number of weeks postpartum, age, and parity, BMI increased more in South Asians than in Europeans $\left(1.5(1.2-1.8) \mathrm{kg} / \mathrm{m}^{2}\right.$ vs 0.1 ( -0.1 to 0.3$) \mathrm{kg} / \mathrm{m}^{2}$ ) (Fig. 3). Among the skinfold measurements, triceps (3.4 (2.4-4.4) $\mathrm{mm}$ vs $1.0(0.3-1.7)$ $\mathrm{mm})$, subscapular (4.0 (2.9-5.1) $\mathrm{mm}$ vs $2.3(1.5-3.1) \mathrm{mm})$, suprailiac (2.7 (1.3-4.0) $\mathrm{mm}$ vs 0.5 ( -0.5 to 1.5$) \mathrm{mm}$ ), and sum of skinfolds (10.0 (7.4-12.7) $\mathrm{mm}$ vs $3.8(1.9-5.8) \mathrm{mm})$ increased more in South Asians than Europeans, after adjustment for weeks of gestation at inclusion, week after

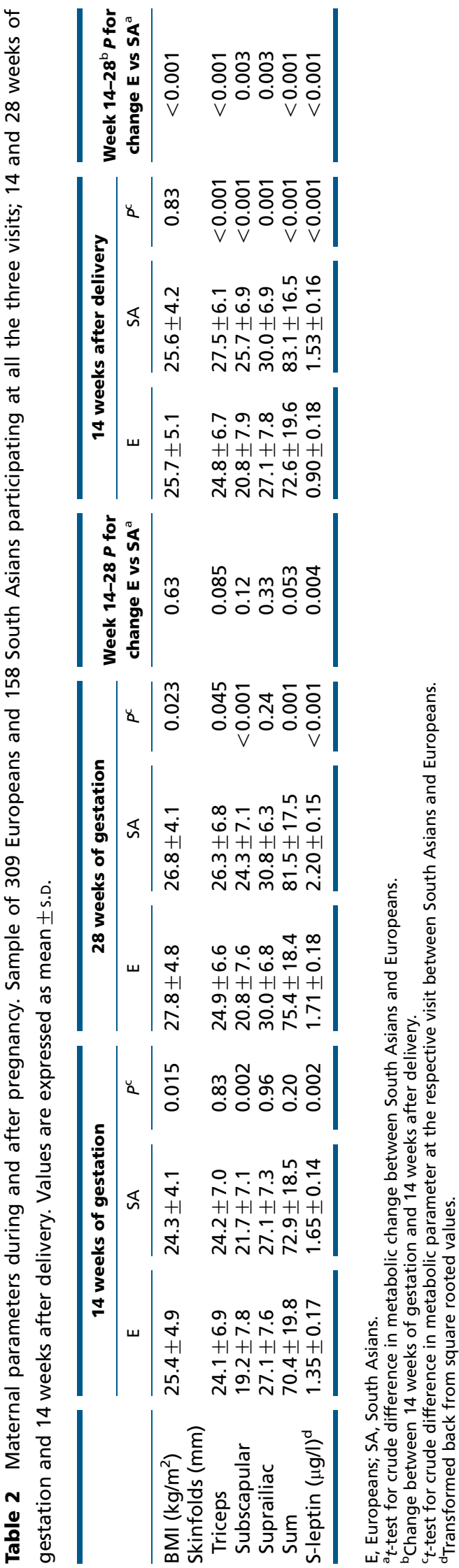



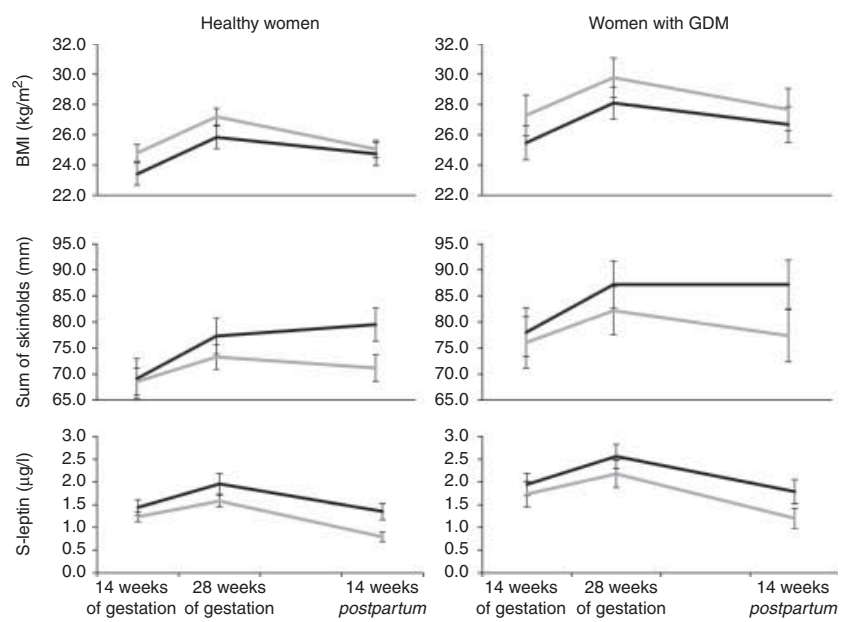

\section{Figure 2}

Longitudinal development of BMI, sum of skinfolds, and s-leptin levels in South Asians (black) and Europeans (gray) in healthy women (left) vs women diagnosed with GDM at 28 weeks of gestation (right). Values are mean and $95 \% \mathrm{Cl}$. S-leptin levels were square rooted to calculate the mean and $95 \% \mathrm{Cl}$ and subsequently transformed back to normal values.

delivery, age, and parity (Fig. 3). S-leptin levels decreased more in Europeans $(-0.47(-0.57$ to 0.37$) \mu \mathrm{g} / \mathrm{l})$ than in South Asians ( $-0.13(-0.27$ to -0.00$) \mu \mathrm{g} / \mathrm{l})$ after adjustment for weeks of gestation at inclusion, week after delivery, age, and parity (Fig. 3).

\section{Effect of parity on ethnic differences in BMI, subcutaneous fat, and s-leptin}

As South Asians retained more weight and fat after pregnancy than Europeans, we hypothesized that this increase in fat may influence metabolic factors in subsequent pregnancies. We therefore explored ethnic differences in body composition at 14 weeks of gestation in nulliparous vs parous women. Among nulliparous women, South Asians had lower BMI than Europeans, while there were no ethnic differences in triceps, subscapular, or suprailiac skinfolds; sum of skinfolds; or s-leptin (Table 3). Among parous women, however, South Asians and Europeans had similar BMI, but South Asians had larger triceps and subscapular skinfolds, larger sum of skinfolds, and higher s-leptin than Europeans (Table 3).

\section{Associations with GDM}

The prevalence of GDM was 23.8\% $(n=84)$ in Europeans and $42.6 \%(n=81)$ in South Asians. Women with GDM had consistently higher BMI, skinfolds, and s-leptin levels than healthy women (Fig. 2). In simple logistic regression analyses, BMI, sum of skinfolds, and s-leptin levels in early pregnancy were all positively associated with GDM (OR (95\% CI); 1.09 (1.04-1.13) per $\mathrm{kg} / \mathrm{m}^{2}$ increase in BMI, 1.02 (1.01-1.03) per $\mathrm{mm}$ increase in sum of skinfolds, and 3.70 (2.29-5.98) per square root increase in s-leptin). In multiple logistic regression analyses (performed separately for each explanatory variable), BMI, sum of skinfolds, and s-leptin remained associated with GDM after adjustments for weeks of gestation at inclusion, age, parity, and ethnic origin (1.10 (1.06-1.15) per $\mathrm{kg} / \mathrm{m}^{2}$ increase in BMI, 1.02 (1.01-1.03) per $\mathrm{mm}$ increase in sum of skinfolds, and 3.31 (2.02-5.43) per square root increase in s-leptin). Parity had no effect on the associations between BMI, subcutaneous fat, or s-leptin and GDM (no significant interaction). Additional adjustment for family history of diabetes did not change the results.

Neither BMI, sum of skinfolds, nor s-leptin completely explained the higher GDM risk observed in South Asians than in Europeans. South Asians had 2.45 (1.63-3.67) higher odds of GDM than Europeans after adjustments for weeks of gestation, age, and parity. Additional adjustment for BMI increased the odds of GDM in South Asians to 2.80 (1.85-4.27) compared with Europeans, while the OR was reduced to 2.19 (1.43-3.34) after adjustment for sum of skinfolds and to 2.07 (1.37-3.14) after adjustment for s-leptin. Sum of skinfolds and s-leptin at 14 weeks of gestation, thereby explained some, but not all, of the ethnic difference in GDM risk.

\section{Discussion}

In this population-based prospective cohort study, South Asians had larger subscapular skinfold, similar triceps and suprailiac skinfold, and higher s-leptin levels in early

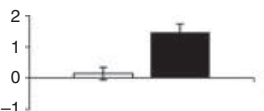

BMI $\left(\mathrm{kg} / \mathrm{m}^{2}\right), P<0.001$

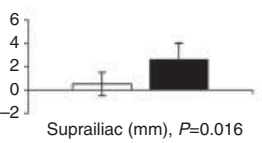

Suprailiac $(\mathrm{mm}), P=0.016$
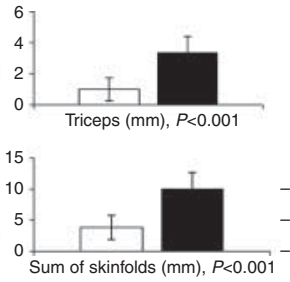

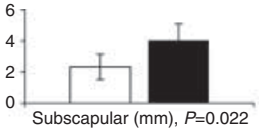

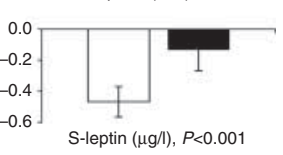

\section{Figure 3}

Change in parameters from 14 weeks of gestation to 14 weeks after delivery. Multivariate general linear models adjusted for weeks of gestation at inclusion, week after delivery, age, and parity. White bars are Europeans, black bars are South Asians. 
Table 3 Effect of parity on ethnic differences in BMI, subcutaneous fat, and s-leptin at 14 weeks of gestation. Values are mean (95\% Cls), adjusted for pregnancy week at measurement, and age.

\begin{tabular}{|c|c|c|c|c|c|c|}
\hline & \multicolumn{3}{|c|}{ Nulliparous } & \multicolumn{3}{|c|}{ Parous } \\
\hline & Europeans $n=172$ & South Asians $n=74$ & $P$ ethnic ${ }^{a}$ & Europeans $n=156$ & South Asians $n=99$ & $P$ ethnic ${ }^{a}$ \\
\hline BMI $\left(k g / m^{2}\right)$ & $25.3(24.6-26.0)$ & $23.4(22.3-24.5)$ & 0.008 & $25.5(24.8-26.2)$ & $25.2(24.3-26.1)$ & 0.59 \\
\hline Triceps (mm) & $23.9(22.8-25.0)$ & $23.1(21.4-24.7)$ & 0.41 & $23.1(22.0-24.2)$ & $26.3(24.9-27.7)$ & 0.001 \\
\hline Subscapular (mm) & $18.9(17.8-20.1)$ & $21.1(19.3-22.9)$ & 0.050 & $19.2(18.0-20.4)$ & $23.1(21.6-24.6)$ & $<0.001$ \\
\hline Suprailiac (mm) & $27.0(25.9-28.1)$ & $27.6(25.8-29.3)$ & 0.60 & $26.2(25.0-27.4)$ & $27.6(26.1-29.1)$ & 0.16 \\
\hline Sum of skinfolds (mm) & $69.8(66.9-72.7)$ & $71.7(67.2-76.2)$ & 0.50 & $68.5(65.4-71.6)$ & $78.0(73.1-80.9)$ & 0.001 \\
\hline S-leptin $(\mu \mathrm{g} / \mathrm{l})^{\mathrm{b}}$ & $1.42(1.28-1.56)$ & $1.51(1.30-1.74)$ & 0.45 & $1.21(1.08-1.35)$ & $1.88(1.66-2.10)$ & $<0.001$ \\
\hline
\end{tabular}

a $P$ for ethnic difference among nulliparous or parous women.

${ }^{\mathrm{b}}$ Transformed back from square rooted values.

pregnancy compared with Europeans, despite having a lower BMI. At 14 weeks after delivery, South Asians had retained more weight and subcutaneous fat than Europeans, and s-leptin level was less reduced in South Asians compared with Europeans. Accordingly, parous South Asians had more subcutaneous fat and s-leptin than parous Europeans, while we did not find corresponding ethnic differences among nulliparous. BMI, subcutaneous fat, and s-leptin were all positively associated with GDM. Some of the ethnic difference in GDM risk was explained by skinfolds and s-leptin, but not by BMI. The higher retention of weight and subcutaneous fat in South Asians may increase their risk of overweight, obesity, and GDM in future pregnancies.

Our finding that South Asians had relatively high amounts of subcutaneous fat, despite having lower BMI than Europeans, is in line with other studies $(24,25)$. Further, the higher relative adiposity in South Asians might be present already at birth, as South Asian neonates have lower birth weight than European, but similar skinfold thickness and cord leptin levels $(21,26,27)$. Supportive of these findings are also the higher leptin levels observed in adult South Asians in several studies $(16,17,28)$.

The South Asians in our sample had similar suprailiac and tricep skinfolds and larger subscapular skinfolds than the Europeans in early pregnancy and the levels increased during and after pregnancy, suggesting that South Asians were capable of storing subcutaneous fat. Subcutaneous fat has been considered a healthy way of storing fat, as it may work as a 'metabolic sink' that buffers energy overflow (29). According to the 'adipose tissue overflow hypothesis' (30), South Asians have a lower capacity to store superficial subcutaneous fat, resulting in deposition of visceral fat when facing energy excess. The higher amount of metabolically active visceral fat has been proposed as one reason why South Asians have a higher diabetes risk than white Europeans (31), despite having the same BMI (32).
Since skinfold measurements are considered crude measures of subcutaneous fat (33), we cannot refute this hypothesis. Also, the suprailiac and subscapular skinfolds have been found to reflect visceral adiposity (34). However, our findings are in accordance with a study that found no differences in deep or superficial subcutaneous fat between Norwegian and Pakistani women with type 2 diabetes (35). On the other hand, South Asians have been found to have larger adipocyte size (36), which, in turn, has been associated with insulin resistance and hyperleptinemia (37) and type 2 diabetes independently of insulin resistance (38). Therefore, the relative amount of subcutaneous fat may be the same, while the number of adipocytes may be lower and the adipocytes larger in South Asians vs Europeans. This could imply that South Asians may have adipocytes that are more metabolically active.

Parous South Asians had higher BMI, skinfolds, and s-leptin levels than parous Europeans, while we did not find the same in nulliparous. This supports our finding of higher weight and subcutaneous fat retention in South Asians than Europeans. We were not able to find any previous studies exploring the effect of parity in relation to weight and subcutaneous fat retention across ethnic origin. Parity has been associated with a decrease in lower body fat and an increase in central fat depots (39). Possible explanations for the higher retention in South Asians may be cultural differences in physical activity and diet in general, particularly in the postpartum period (40). Weight gain during pregnancy was a strong predictor of weight retention after delivery in our cohort (40). Future studies should explore possible reasons for the higher postpartum weight retention in South Asians.

As expected, BMI, subcutaneous fat, and s-leptin levels in early pregnancy were all associated with the risk of GDM both before and after adjusting for covariates. Although s-leptin seemed to be the most important of these, it did not entirely explain the higher risk of GDM in South Asians. 
Strengths of the present study include high participation rate, low risk of selection bias, and follow-up after delivery. Limitations of our study include the small difference between the ethnic groups in median weeks of gestation at inclusion, and this was adjusted for in the analyses. Also, assessment of regional fat distribution was limited to the measurement of skinfold thickness, as assessment with $\mathrm{X}$-ray-based methods is considered inappropriate due to radiation hazard in pregnancy, and magnetic resonance imaging was not possible in a community-based, epidemiological study like this. Some of the raters had relatively high inter-rater variability on a few measurements, but the overall inter-rater variability was considered adequate for use at a group level. The use of sum of skinfolds instead of each skinfold separately has been found to even out potential bias (41). Although we found no indication of bias related to the inter-rater variability, the presence of random errors may increase the risk of false negative findings.

In conclusion, South Asians had more subcutaneous fat and higher s-leptin levels, despite having lower BMI, than Europeans in early pregnancy, and both subcutaneous fat and s-leptin in early pregnancy were associated with the risk of GDM. Despite similar gain of subcutaneous fat between 14 and 28 weeks of gestation, South Asians retained more subcutaneous fat after delivery, leaving them more adipose when entering future pregnancies. Health professionals should put more emphasis on prevention of excessive weight gain during pregnancy and maternal weight retention after delivery.

\section{Declaration of interest}

The authors declare that there is no conflict of interest that could be perceived as prejudicing the impartiality of the research reported.

\section{Funding}

The STORK Groruddalen study was funded by the Norwegian Research Council, the Norwegian Directorate of Health, and the South Eastern Norway Regional Health Authority.

\section{Author contribution statement}

C Sommer designed the substudy, performed all statistical analyses, and drafted and edited the manuscript. A K Jenum initiated and was the project leader of the STORK Groruddalen study. K Mørkrid prepared the glucose data for the analysis. L Sletner and K Mørkrid participated in the quality control of the data. K I Birkeland designed the substudy, contributed to the conception and design of the study, and is the leader of the study's steering committee. C Sommer, A K Jenum, K Mørkrid, L Sletner, C W Waage, and $\mathrm{K}$ I Birkeland contributed to the interpretation of the data, discussions, critical revision of the manuscript, and have approved the final version of the manuscript.

\section{Acknowledgements}

The authors thank midwives and research staff at Grorud, Bjerke, and Stovner child health clinics and the women who participated in the STORK Groruddalen study.

\section{References}

1 WHO. Global Status Report on Noncommunicable Diseases 2010. Geneva: World Health Organization, 2011.

2 American Diabetes Association. Economic costs of diabetes in the U.S in 2012. Diabetes Care 201336 1033-1046. (doi:10.2337/dc12-2625)

3 Tillin T, Hughes AD, Godsland IF, Whincup P, Forouhi NG, Welsh P, Sattar N, McKeigue PM \& Chaturvedi N. Insulin resistance and truncal obesity as important determinants of the greater incidence of diabetes in Indian Asians and African Caribbeans compared with Europeans: the Southall And Brent REvisited (SABRE) cohort. Diabetes Care 201336 383-393. (doi:10.2337/dc12-0544)

4 Jenum AK, Mørkrid K, Sletner L, Vangen S, Torper JL, Nakstad B, Voldner N, Rognerud-Jensen OH, Berntsen S, Mosdøl A et al. Impact of ethnicity on gestational diabetes identified with the WHO and the modified International Association of Diabetes and Pregnancy Study Groups criteria: a population-based cohort study. European Journal of Endocrinology 2012166 317-324. (doi:10.1530/EJE-11-0866)

5 O'Sullivan JB, Gellis SS, Dandrow RV \& Tenney BO. The potential diabetic and her treatment in pregnancy. Obstetrics and Gynecology 1966 27 683-689.

6 Bellamy L, Casas JP, Hingorani AD \& Williams D. Type 2 diabetes mellitus after gestational diabetes: a systematic review and meta-analysis. Lancet 2009373 1773-1779. (doi:10.1016/S01406736(09)60731-5)

7 Benhalima K, Hanssens M, Devlieger R, Verhaeghe J \& Mathieu C. Analysis of pregnancy outcomes using the new IADPSG recommendation compared with the carpenter and Coustan criteria in an area with a low prevalence of gestational diabetes. International Journal of Endocrinology 20132013 248121. (doi:10.1155/2013/248121)

8 Wendland EM, Torloni MR, Falavigna M, Trujillo J, Dode MA, Campos MA, Duncan BB \& Schmidt MI. Gestational diabetes and pregnancy outcomes - a systematic review of the World Health Organization (WHO) and the International Association of Diabetes in Pregnancy Study Groups (IADPSG) diagnostic criteria. BMC Pregnancy and Childbirth 201212 23. (doi:10.1186/1471-2393-12-23)

9 Dabelea D. The predisposition to obesity and diabetes in offspring of diabetic mothers. Diabetes Care 200730 S169-S174. (doi:10.2337/ dc07-s211)

10 Surkan PJ, Hsieh CC, Johansson AL, Dickman PW \& Cnattingius S. Reasons for increasing trends in large for gestational age births. Obstetrics and Gynecology 2004104 720-726. (doi:10.1097/01.AOG. 0000141442.59573.cd)

11 Aune D, Saugstad OD, Henriksen T \& Tonstad S. Maternal body mass index and the risk of fetal death, stillbirth, and infant death: a systematic review and meta-analysis. Journal of the American Medical Association 2014311 1536-1546. (doi:10.1001/jama.2014.2269)

12 Kahn SE, Hull RL \& Utzschneider KM. Mechanisms linking obesity to insulin resistance and type 2 diabetes. Nature $2006 \mathbf{4 4 4} 840-846$. (doi:10.1038/nature05482)

13 Frayn KN, Karpe F, Fielding BA, Macdonald IA \& Coppack SW. Integrative physiology of human adipose tissue. International Journal of Obesity and Related Metabolic Disorders 200327 875-888. (doi:10.1038/ sj.ijo.0802326)

14 Minocci A, Savia G, Lucantoni R, Berselli ME, Tagliaferri M, Calo G, Petroni ML, de Medici C, Viberti GC \& Liuzzi A. Leptin plasma concentrations are dependent on body fat distribution in obese 
patients. International Journal of Obesity and Related Metabolic Disorders 200024 1139-1144. (doi:10.1038/sj.ijo.0801385)

15 Van Harmelen V, Reynisdottir S, Eriksson P, Thorne A, Hoffstedt J, Lonnqvist F \& Arner P. Leptin secretion from subcutaneous and visceral adipose tissue in women. Diabetes 199847 913-917. (doi:10.2337/ diabetes.47.6.913)

16 Mente A, Razak F, Blankenberg S, Vuksan V, Davis AD, Miller R, Teo K, Gerstein $\mathrm{H}$, Sharma AM, Yusuf $\mathrm{S}$ et al. Ethnic variation in adiponectin and leptin levels and their association with adiposity and insulin resistance. Diabetes Care 201033 1629-1634. (doi:10.2337/dc09-1392)

17 Lilja M, Rolandsson O, Shaw JE, Pauvaday V, Cameron AJ, Tuomilehto J, Alberti KG, Zimmet PZ \& Soderberg S. Higher leptin levels in Asian Indians than Creoles and Europids: a potential explanation for increased metabolic risk. International Journal of Obesity 201034 878-885. (doi:10.1038/ijo.2010.19)

18 Tessier DR, Ferraro ZM \& Gruslin A. Role of leptin in pregnancy: consequences of maternal obesity. Placenta 201334 205-211. (doi:10.1016/j.placenta.2012.11.035)

19 Jenum AK, Sletner L, Voldner N, Vangen S, Mørkrid K, Andersen LF, Nakstad B, Skrivarhaug T, Rognerud-Jensen OH, Roald B et al. The STORK Groruddalen research programme: a population-based cohort study of gestational diabetes, physical activity, and obesity in pregnancy in a multiethnic population. Rationale, methods, study population, and participation rates. Scandinavian Journal of Public Health 201038 60-70. (doi:10.1177/1403494810378921)

20 Sommer C, Mørkrid K, Jenum AK, Sletner L, Mosdøl A \& Birkeland KI. Weight gain, total fat gain and regional fat gain during pregnancy and the association with gestational diabetes: a population-based cohort study. International Journal of Obesity 201438 76-81. (doi:10.1038/ ijo.2013.185)

21 Sletner L, Nakstad B, Yajnik CS, Morkrid K, Vangen S, Vardal MH, Holme IM, Birkeland KI \& Jenum AK. Ethnic differences in neonatal body composition in a multi-ethnic population and the impact of parental factors: a population-based cohort study. PLOS ONE $2013 \mathbf{8}$ e73058. (doi:10.1371/journal.pone.0073058)

22 Brannsether B, Roelants M, Bjerknes R \& Juliusson PB. References and cutoffs for triceps and subscapular skinfolds in Norwegian children 4-16 years of age. European Journal of Clinical Nutrition 2013 67 928-933. (doi:10.1038/ejcn.2013.91)

23 WHO Guidelines Approved by the Guidelines Review Committee. Diagnostic Criteria and Classification of Hyperglycaemia First Detected in Pregnancy. Geneva: World Health Organization, 2013.

24 Lear SA, Kohli S, Bondy GP, Tchernof A \& Sniderman AD. Ethnic variation in fat and lean body mass and the association with insulin resistance. Journal of Clinical Endocrinology and Metabolism 200994 4696-4702. (doi:10.1210/jc.2009-1030)

25 Dudeja V, Misra A, Pandey RM, Devina G, Kumar G \& Vikram NK. BMI does not accurately predict overweight in Asian Indians in northern India. British Journal of Nutrition 200186 105-112. (doi:10.1079/BJN2001382)

26 Yajnik CS, Lubree HG, Rege SS, Naik SS, Deshpande JA, Deshpande SS, Joglekar CV \& Yudkin JS. Adiposity and hyperinsulinemia in Indians are present at birth. Journal of Clinical Endocrinology and Metabolism 2002 87 5575-5580. (doi:10.1210/jc.2002-020434)

27 West J, Lawlor DA, Fairley L, Bhopal R, Cameron N, McKinney PA, Sattar N \& Wright J. UK-born Pakistani-origin infants are relatively more adipose than white British infants: findings from 8704 motheroffspring pairs in the Born-in-Bradford prospective birth cohort. Journal of Epidemiology and Community Health 201367 544-551. (doi:10.1136/jech-2012-201891)
28 Shah A, Hernandez A, Mathur D, Budoff MJ \& Kanaya AM. Adipokines and body fat composition in South Asians: results of the Metabolic Syndrome and Atherosclerosis in South Asians Living in America (MASALA) study. International Journal of Obesity 201236 810-816. (doi:10.1038/ijo.2011.167)

29 Despres JP \& Lemieux I. Abdominal obesity and metabolic syndrome. Nature 2006444 881-887. (doi:10.1038/nature05488)

30 Sniderman AD, Bhopal R, Prabhakaran D, Sarrafzadegan N \& Tchernof A. Why might South Asians be so susceptible to central obesity and its atherogenic consequences? The adipose tissue overflow hypothesis. International Journal of Epidemiology 200736 220-225. (doi:10.1093/ije/dyl245)

31 Bhopal RS. A four-stage model explaining the higher risk of type 2 diabetes mellitus in South Asians compared with European populations. Diabetic Medicine 201330 35-42. (doi:10.1111/dme.12016)

32 Boffetta P, McLerran D, Chen Y, Inoue M, Sinha R, He J, Gupta PC, Tsugane S, Irie F, Tamakoshi A et al. Body mass index and diabetes in Asia: a cross-sectional pooled analysis of 900,000 individuals in the Asia cohort consortium. PLOS ONE 20116 e19930. (doi:10.1371/journal. pone.0019930)

$33 \mathrm{Hu}$ FB. Measurement of adiposity and body composition. In Obesity Epidemiology, ch 5, pp 53-83. Ed. FB Hu. New York, NY: Oxford University Press, Inc., 2008.

34 Ganpule-Rao A, Joglekar C, Patkar D, Chinchwadkar M, Bhat D, Lubree H, Rege S, Uradey B, Yajnik C \& Yudkin J. Associations of trunk fat depots with insulin resistance, $\beta$ cell function and glycaemia $-\mathrm{a}$ multiple technique study. PLOS ONE 20138 e75391. (doi:10.1371/ journal.pone.0075391)

35 Wium C, Eggesbo HB, Ueland T, Michelsen AE, Torjesen PA, Aukrust P $\&$ Birkeland K. Adipose tissue distribution in relation to insulin sensitivity and inflammation in Pakistani and Norwegian subjects with type 2 diabetes. Scandinavian Journal of Clinical and Laboratory Investigation 201474 700-707. (doi:10.3109/00365513.2014.953571)

36 Anand SS, Tarnopolsky MA, Rashid S, Schulze KM, Desai D, Mente A, Rao S, Yusuf S, Gerstein HC \& Sharma AM. Adipocyte hypertrophy, fatty liver and metabolic risk factors in South Asians: the Molecular Study of Health and Risk in Ethnic Groups (mol-SHARE). PLoS ONE 20116 e22112. (doi:10.1371/journal.pone.0022112)

37 Lundgren M, Svensson M, Lindmark S, Renstrom F, Ruge T \& Eriksson JW. Fat cell enlargement is an independent marker of insulin resistance and 'hyperleptinaemia'. Diabetologia 200750 625-633. (doi:10.1007/s00125-006-0572-1)

38 Weyer C, Foley JE, Bogardus C, Tataranni PA \& Pratley RE. Enlarged subcutaneous abdominal adipocyte size, but not obesity itself, predicts type II diabetes independent of insulin resistance. Diabetologia 200043 1498-1506. (doi:10.1007/s001250051560)

39 Lassek WD \& Gaulin SJ. Changes in body fat distribution in relation to parity in American women: a covert form of maternal depletion. American Journal of Physical Anthropology 2006131 295-302. (doi:10.1002/ajpa.20394)

40 Waage C, Falk R, Sommer C, Morkrid K, Richardsen K, Baerug A, Shakeel N, Birkeland K \& Jenum A. Ethnic differences in postpartum weight retention: a Norwegian cohort study. BJOG: an International Journal of Obstetrics and Gynaecology 2015. In press. (doi:10.1111/ 1471-0528.13321)

41 Ulijaszek SJ \& Kerr DA. Anthropometric measurement error and the assessment of nutritional status. British Journal of Nutrition $1999 \mathbf{8 2}$ 165-177. (doi:10.1017/S0007114599001348)

Received 16 January 2015

Revised version received 2 March 2015

Accepted 4 March 2015 